\section{SOME NOTES ON THE BACTERIOLOGY OF GAS GANGRENE.}

By ALEXANDER FLEMING, F.R.C.S. ENG., ASSISTANT LEOTURER IN BAOTERIOLOGY, ST. MARY'S HOSPITAL, AND PATHOLOGIST TO 'IHE LONDON LOCK HOSPITALS.

(From the Research Laboratory attached to No. 13 General Hospital, Boulogne.)

THIS paper is based on observations made in the latter months of 1914 on cases of wounded soldiers in the hospitals in Boulogne, where $I$ had the opportunity of studying these cases in Sir Almroth Wright's research laboratory. We have bacteriological records of 32 cases of so-called gas gangrene or gas phlegmon, and we have examined microscopically materials from a considerable number of other cases of the same nature. The bacteriology of all these was, however, essentially the same, so I will confine myself to the first series.

TABLE I.-Cases of Gas Gangrene.

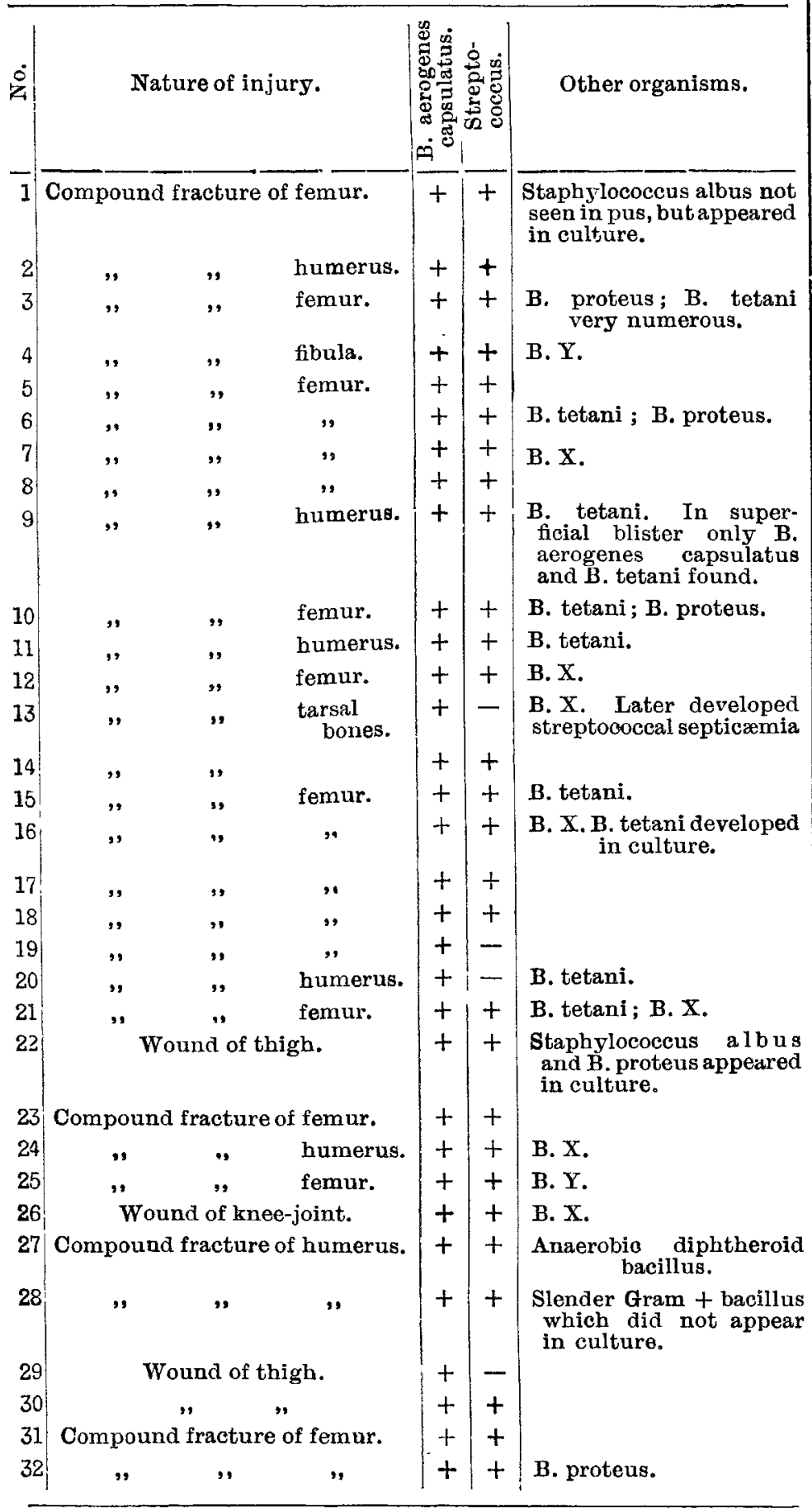

In this table I have named two of the bacilli found " $\mathrm{X}$ " and "Y." These are both spore-bearing anaerobic bacilli which are responsible largely for the foul smell of the gangrenous wounds. I have been unable to demonstrate any pathogenic action of these bacilli. The bacteriological findings are set forth above in tabular form. In every case we found the dominant organism to be $B$. aerogenes capsulatus. Rarely it was apparently in pure culture. Commonly it was associated with streptococci and frequently other organisms were present.

In the very numerous class where only B. aerogenes capsulatus and streptococci were present the gas production must clearly have been due to the former, as we know well that streptococcus is incapable of yas production in tissues. We shall see later, however, that the streptococcus aids B. aerogenes capsulatus as a gas producer in blood serum. Again, B. aerogenes capsulatus was the only one of the organisms which we have isolated from these gangrene cases which was capable of reproducing the condition in animals. There seems to be no doubt that it is this organism which was responsible for the emphysema of the tissues in these cases. We have not been able to discover the bacillus of malignant cedema (vibrion septique) in any one of the cases examined.

In all the cases in which I saw gas gangrene develop the wound was situated in the upper or lower extremity, and much more commonly in the latter. I have not seen the condition follow a wound of the trunk. Again, wounds in which the B. aerogenes capsulatus exercised its gas-producing properties were those in which the drainage was deficient, so that there resulted a gross infection of the imprisoned discharges which ultimately proved too strong for the resistance of the tissues, and resolved itself into an infection of the tissues themselves with the gas-producing organism. In some of the cases the gangrenous condition of the limb resulted from the mechanical obstruction of the circulation due to interference with the arterial channels of the limb, but in others the blockage of the artery was apparently the result of a septic process spreading to the vessel. The bacteria found depended to a certain extent on the portion of the limb from which the material for examination was obtained.

When material from the wounds was examined it was usual to find $B$. aerogenes and streptococcus with putrefactive spore-bearing organisms and frequently B. proteus. (Fig. 1.) If, however, the cultures were made from a portion of the gangrenous limb away from the wound, then B. aerogenes capsulatus alone or combined with streptococcus was the common finding.

On several occasions I examined films made from different portions of a limb amputated for gas gangrene and $I$ found large numbers of $B$. aerogenes capsulatus in every plane from the skin to the bone marrow. They were especially numerous in the fascial planes between the muscles and in the subcutaneous tissue. Usually there was a fair amount of phagocytosis of these organisms, indicating an attempt at resistance to the infection, even after the limb had become gangrenous.

\section{Description of B. Aerogenes Capsulatus.}

This is a large, straight, non-motile anaerobic bacillus, with almost square ends. In the body it measures about $5 \mu \times 2 \mu$, but in culture the length varies enormously when different media are used. It is very strongly Gram-positive. Sometimes, both in material from the body and in serum culture, ordinary methods of staining show it to possess a very definite capsule (Fig. 2), but more often the capsule is not evident. 
FIG. 1.

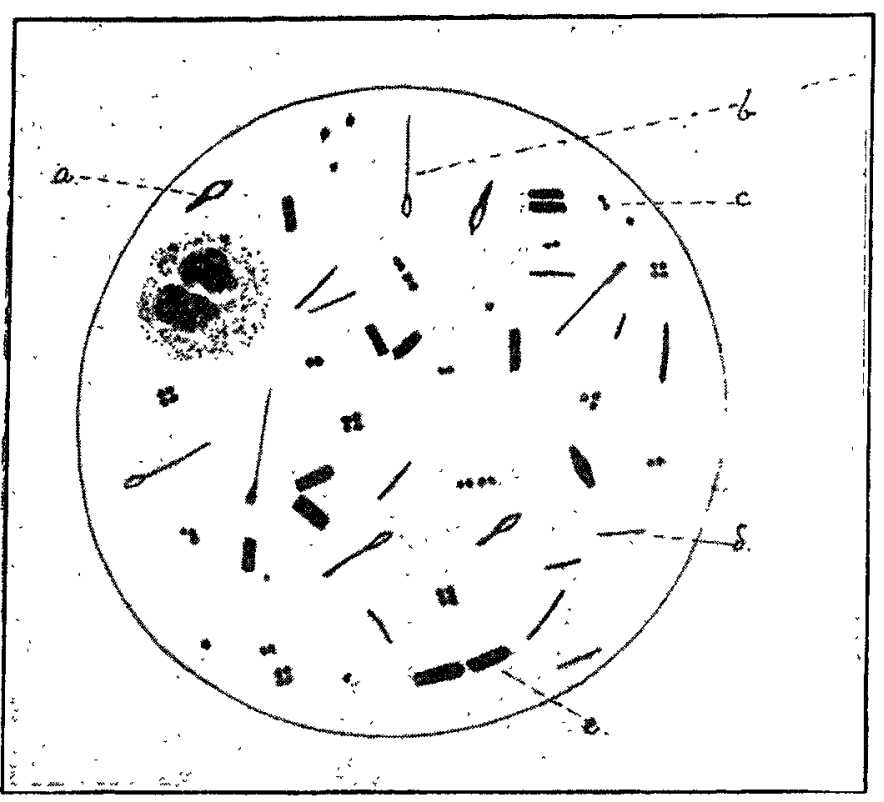

Film from wound in a case of gas gangrene. A, B. X. B, B. Y. c, Streptococeus. D, B. proteus. E, B. aerogenes capsulatus.

Spore formation.-In the body it forms spores which are, as a rule, situated near one end. The spores are large and oval, and distort the bacillus. Occasionally a median spore is seen. (Fig. 3.) In culture spores are only seen in special conditions; on sugar media they do not occur; in broth spores are occasionally seen; in cultures in blood serum they are usually to be found in very small numbers; and on neutral-red egg medium, although I could not detect spores microscopically, I found that a temperature of $80^{\circ} \mathrm{C}$. for one hour would not sterilise cultures of more than two days' growth. Douglas found that if this bacillus is grown in undiluted human blood serum anaerobically with staphylococcus in two days very copious sporing occurs. By taking advantage of this

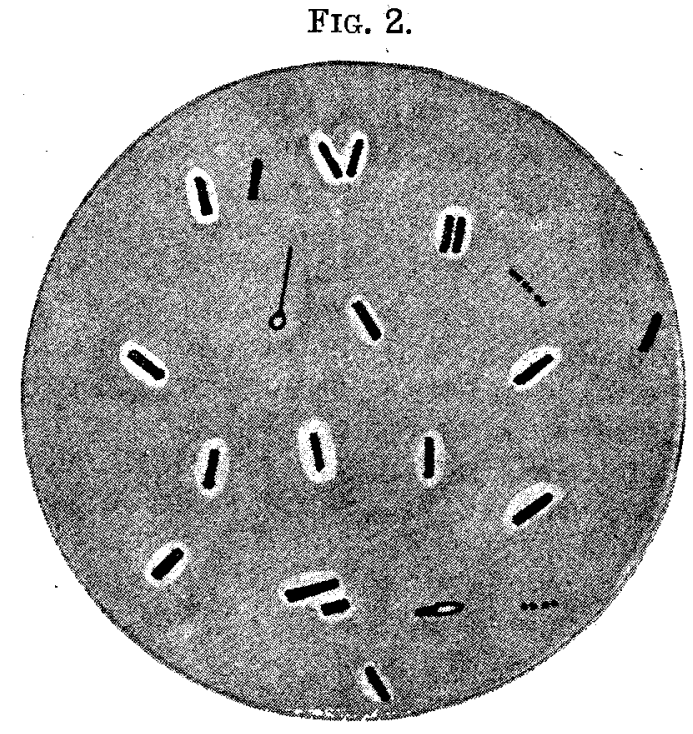

Film of fluid from vesicle on a gangrenous limb, showing B. aerogenes capsulatus, $B$, tetani, and streptococei. one can easily separate $B$. aerogenes capsulatus from a culture containing non-sporing bacteria. The mixed culture is planted anaerobically with staphylococcus into human blood serum, and after two or three days the culture is heated to $80^{\circ} \mathrm{C}$. for half an hour and planted into milk.

Isolation from the body.-The easiest way is to boil a tube of sterile milk, and immediately while it is yet at a temperature close to $100^{\circ} \mathrm{C}$. plant into it some of the pus. This kills the non-sporing bacteria, and (unless other spore-bearing bacilli were present) a pure culture of B. aerogenes capsulatus results. If other sporing bacteria are present then one has either to separate by plating or by planting into agar or glucose agar and drawing this up into a Vignal's tube. The separate colonies which develop can then be dealt with.

The anaerobicity of this organism is not very strict, and it was found quite sufficient to use tubes of milk containing about 10 c.c. and to incubate them without anaerobic precautions. The scum of cream which formed in such a tube was of considerable thickness, and apparently it shut off the air to a sufficient extent.
Cultural Characteristics.

from six hours upwards very firm clotting occurs with the evolution of much gas. the appearance and staining reactions are Agar.-In stab or shake culture there is growth almost to the surface and much gas

Glucose agar.-For surface colonies the anaerobic conditions have to be perfect. The colonies are Blood glucose agar (glucose agar with one-tenth part of blood added).-This is a very favourable medium for surface growth. The colonies were large, round, greyish, and very flat. The bacilli on this medium showed very long forms.

Neutral-red egg medium.This is the best solid medium for surface growth of the bacillus. In 24 hours these colonies appear as bright pink spots about $2 \mathrm{~mm}$. in size. They have a slightly raised margin and show a very characteristic prominence in their centre. The bacilli on this medium are very short and stout, many of them being little longer than they are broad.

Pathogenicity for guineapigs.-1 c.c. of a 24 -hours' milk culture was injected into the hind leg of a guinea-pig subcutaneously. In 12 hours there was a swelling extending to the axilla on both sides. Death resulted in 24 hours. On

FIG. 3.

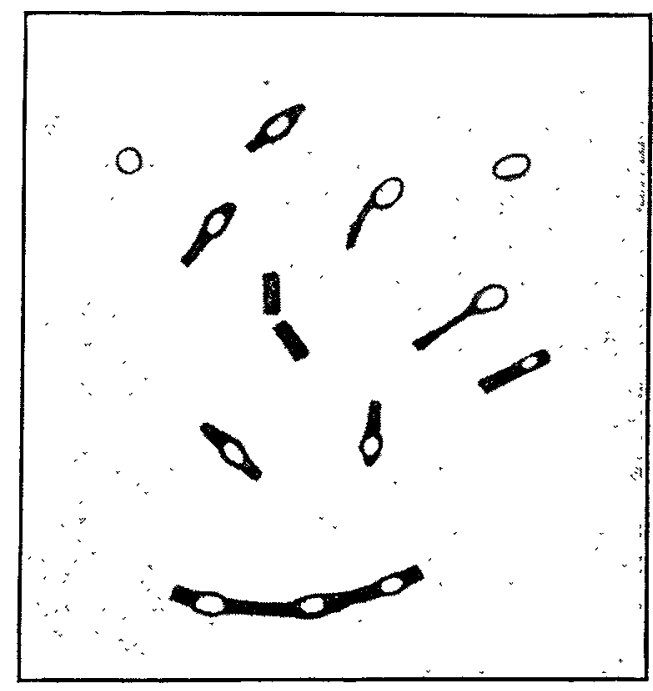

B. aerogenes capsulatus. Sporing forms observed in culture with staphylococcus in serum. 
opening the animal it was found that the whole of the skin had been ripped off the underlying muscles by gas and a serous exudate. The muscles of the thigh and the pectoral muscles on both sides looked as if they had been dissected out, and were bathed in the sero-gaseous effusion. The internal organs were congested; the liver was a little larger than normal; the spleen was normal in size but very dark in colour. The bacillus could be seen in considerable numbers in the heart's blood and in 3 smears from all the organs. The serous exudate 4 in the subcutaneous tissue of the abdomen contained very large numbers of the bacilli, some of them showing sporing forms. 1/10 c.c. of culture in serum injected subcutaneously into guineapigs was usually fatal, but in one case there resulted intense œdema with gas formation in the subcutaneous tissues, which progressed for about 36 hours and then gradually disappeared. This was very remarkable, as the extent of the gas phlegmon and the extreme severity of the symptoms rendered the chances of recovery of the guinea-pig apparently hopeless. This guinea-pig by its complete recovery showed that even when the infection was far advanced an immunity may be developed which will overcome the infection.

B. aerogenes capsulatus is an organism to which there exists in the blood serum normally a very considerable amount of opsonic substance, rendering the organism very easily capable of phagocytosis. It is usual, indeed, to see in the discharges from wounds this bacillus ingested by leucocytes. Possibly this, in part at least, accounts for the relative non-virulence of the bacillus. Its presence in almost all wounds has been demonstrated, but it is only comparatively seldom that it is seen to exercise its full pathogenic action, evidenced by the production of gas gangrene. In such cases there has nearly always been a very considerable amount of dead material or blood clot enclosed in the wound, which furnishes an admirable culture medium for the bacillus. When this bacillus grows on blood it produces a considerable amount of lactic acid, and it has been shown that lactic acid has a very potent action on leucocytes, preventing their emigration. Thus we have a vicious circle; the greater the growth of the bacillus the more is the production of lactic acid, and consequently the less is the leucocytic emigration. It can easily be seen, therefore, that unless such a vicious circle is broken the result is likely to be disastrous. The only efficient way to break it is by means of free drainage, thus diminishing the amount of culture medium for the bacillus and at the same time furnishing an adequate exit for the products of its growth.

It is rare, if not unknown, to see gas gangrene develop in a patient whose wound has been thoroughly opened up and drained. In this connexion it is interesting to note the effect of symbiosis of B. aerogenes capsulatus and other organisms. I have done a number of experiments in which I grew various mixtures of bacteria in human blood serum and watched the rate of growth as evidenced by the amount of gas formation. When staphylococcus was introduced with the bacillus the gas formation was noted much earlier than when the bacillus was grown pure, and at the end of 24 hours the amount of gas produced was much greater. The same thing, but to a lesser extent, was seen when streptococcus or B. proteus was added. We know that staphylococcus, streptococcus, and B. proteus are themselves incapable of producing gas in blood serum. Therefore the increased production must have been due to an increased activity of $B$. aerogenes capsulatus.

TABLE II.-Showing the Effect of Growing B. Aerogenes Capsulatus in Association with other Organisms in Human Blood s'erum.

24 hours.

1. B. aerogenes capsulatus pure $\quad \ldots \quad \ldots \quad$... Small bubble of gas.

$$
\begin{aligned}
& \text { " } \quad \text { " + stapbylococei ... } 1 \text { in. of tube filled with gas, }
\end{aligned}
$$

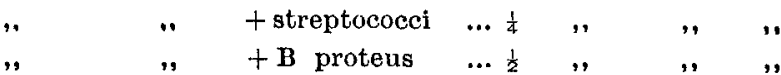

FIG. 4.

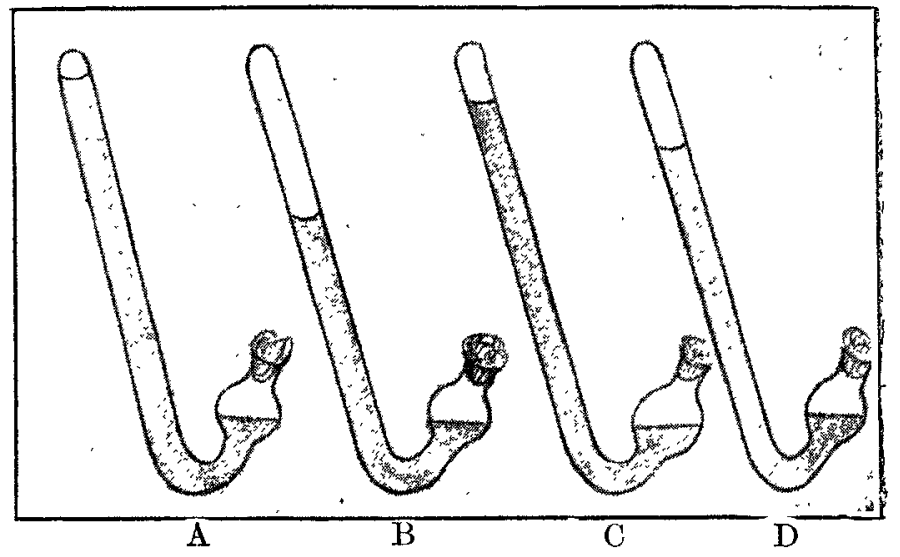

Showing increase in gas formation when B. aerogenes capsulatus is gr swn with other organisms. A, B. aerogenes capsulatus pure B, B. aerogenes capsulatus + staphylo$\mathrm{D}, \mathrm{B}$. aerogenes capsulatus $+\mathrm{B}$. proteus.

This increase of growth of the gas-forming: organisms by association with the common pyogenic bacteria is probably of great importance in the incidence of gas gangrene. The presence of, say, streptococcus aids B. aerogenes capsulatus to establish itself and to produce more rapidly substances which protect the bacillus from the counter attacks of the leucocytes, thus allowing it successfully to storm the defences of the tissues.

As regards the treatment of gas gangrene the main essential, both prophylactically and curatively. is free drainage. The progress of the disease is too rapid to allow of any curative vaccine treatment, but it is possible that if a vaccine of $B$. aerogenes capsulatus and streptococcus were injected very early (in exactly the same way as tetanus antitoxin) the temporary increase in immunity to these organisms. would be sufficient in most cases, at any rate, to prevent the spread of the infection into the tissues. Large doses of $B$. aerogenes capsulatus vaccine (up to $1.000,000,000$ ) can be injected without any reaction and without the development of any "negative phase"; so it would appear that such a vaccine would be perfectly safe to use, and I hope in the near future to see the use of such a vaccine justified by results.

Bristol RoYal InfIRMARY.-The half-yearly meeting of the governors of this infirmary was held on August 10th, under the presidency of Sir George White. The medical report showed that there had been a decrease in the number of in-patients admitted on the civil side of the institution, but during the six months there have been about 2000 military in-patients, and there have never been less than 400 beds occupied in the civil and military sides of the infirmary. The out-patients numbered 21,269 , compared with 17,475 for the corresponding period of 1914. It was stated that the total deficit on revenue amounts to $£ 13,720$, and adding the balance against the charity on the building account, which amounts to £22,904, there is at present a debt of $£ 36,624$ against the in firmary. 